\title{
Some rationalizability results for dynamic games
}

\author{
Ken-Ichi Akao* and Gerhard Sorger** \\ * School of Social Sciences, Waseda University \\ ** Department of Economics, University of Vienna
}

\begin{abstract}
This paper shows that the dynamics generated by a dynamic optimization problem satisfying the standard assumptions of optimal growth theory can also be generated by a symmetric Markovperfect equilibrium of a dynamic game with several identical players. Furthermore, we show that the converse result holds also provided that the equilibrium value functions of the dynamic game are concave.
\end{abstract}

Journal of Economic Literature classification codes: C73, O41, Q50

Key words: Dynamic optimization, dynamic games, rationalizability 


\section{Introduction}

Because rationality is one of the central hypothesis of modern economics, many economic models are formulated as optimization problems or as games. The key difference between these two frameworks is that the former describes the behavior of a single decision maker whereas the latter takes into account the strategic behavior of several interacting agents. An interesting question then is whether these two classes of models generate results that are observationally distinguishable. We address this question in a dynamic context. More specifically, we define two rather general classes of dynamic optimization problems and dynamic games, respectively, and we study whether the dynamics generated by a model of one class can also be generated by models of the other class. Our main findings are that the dynamics generated by a dynamic optimization problem can always be rationalized as the Markov-perfect equilibrium dynamics of a game, and that the converse result holds at least as long as the equilibrium value functions of the dynamic game are concave.

Our study belongs to the economic literature on intertemporal inverse problems or, as they are also called, rationalizability problems. In that literature one seeks to find conditions under which a given outcome (e.g., a dynamical system) can be represented as the solution of an economically meaningful model that satisfies standard assumptions. As for dynamic optimization models satisfying the standard assumptions of optimal growth theory, this literature is quite extensive. The pioneering works are Boldrin and Montrucchio (1986) and Deneckere and Pelikan (1986), and a recent survey is given in Sorger (2006). On the other hand, there are only very few comparable results for dynamic game models. One reason for this is that a dynamic optimization problem can be considered as a dynamic game with a single player. Hence, if a certain dynamical system can be rationalized by a dynamic optimization problem, one can trivially rationalize it also by a dynamic game if the latter either has only a single player or if there are multiple players but only one of them has a non-trivial choice set. So in order to make a rationalizability result for dynamic games interesting, one has to ensure that there really is a non-trivial strategic interaction between several players. Dana and Montrucchio (1986) do that by focusing on duopoly games in which the players make alternating moves. In the present paper we take a different approach, namely we consider simultaneous move games with $n \geq 2$ players and impose symmetry both on the structure of the game 
and on the equilibrium.

The rest of the paper is organized as follows. Section 2 starts by describing the class of dynamic games that we are considering. The games belonging to this class have a natural interpretation as common property resource models but, due to the generality of the specification, many other applications are also possible. The equilibrium concept we are using is Markov-perfect Nash equilibrium (MPNE) which is probably the most popular equilibrium concept for dynamic games of this form. As has been emphasized above, we need to impose some structural assumptions in order to obtain non-trivial rationalizability results. These assumptions are that all players in the game are identical in terms of their preferences and action spaces. Furthermore, we also impose that all of them use the same equilibrium strategies. In other words, we consider only symmetric MPNE in a class of symmetric games. Next, we define a class of optimization problems which can be considered as special cases of the dynamic games that one obtains by assuming that there is only a single player with a linear utility function. This class of dynamic optimization problems basically coincides with the class of optimal growth models that has been studied extensively in the rationalizability literature; see Sorger (2006). Finally, we define the rationalizability problem in the context of the two families of models under consideration.

In Section 3 we show that an optimal path of a dynamic optimization problem can be reproduced as an equilibrium path corresponding to a symmetric MPNE of a dynamic game with $n$ players, where $n \geq 2$ can be chosen arbitrarily (Theorem 3.1). The proof of this result is constructive and highlights the relation between the optimal value function of the optimization problem on the one hand and the equilibrium value function of the game on the other. As a straightforward implication of this theorem and a result by Mitra and Sorger (1999) we can show that, for every Lipschitz continuous function, one can find a dynamic game model for which the given function generates an MPNE path.

The construction used to prove Theorem 3.1 uses utility functions with wealth effects. In Section 4 we study the question of whether it is possible to impose some convenient properties (like monotonicity or concavity) on the wealth effect. We show that this is indeed the case provided that some smoothness properties are satisfied and provided that the discount factor is sufficiently close to zero (Theorem 4.1). A few examples are presented to show that a low discount factor is sufficient for Theorem 4.1 but by far not necessary. 
Finally, in Section 5 we address the problem of proving a partial converse of Theorem 3.1, i.e., we study the rationalizability of an equilibrium path of a dynamic game by a single-player optimization model. This is a more tricky problem because dynamic games are more general than dynamic optimization problems and, consequently, the set of equilibrium paths generated by MPNE is likely to be larger than the set of optimal paths for dynamic optimization problems. We can therefore not expect to get a full converse to Theorem 3.1 but only a partial one. The result that we can prove (Theorem 5.1) makes essentially only one significant structural assumption, namely that the equilibrium value function of the dynamic game is a concave function of the state variables. This property is not true in the general class of dynamic games under consideration because, due to the non-linearity of the equilibrium strategies, each player's optimization problem does not necessarily satisfy the convexity assumptions that are typically used to prove the concavity of the value function.

Some concluding remarks are collected in Section 6.

\section{Definitions and preliminaries}

\subsection{Dynamic games}

Let us denote by $\mathbb{R}$ and $\mathbb{R}_{+}$the set of real numbers and the set of non-negative real numbers, respectively. Furthermore, let $\mathbb{N}$ be the set of positive integers.

Time evolves in discrete periods, i.e., the time variable $t$ takes values in $\mathbb{N}$. We denote by $x_{t-1} \in X \subseteq$ $\mathbb{R}_{+}^{m}$ the vector of capital stocks at the end of period $t-1$ (or, equivalently, at the beginning of period t). The capital stocks may include common property resources such as natural resources and the global atmosphere. The set $X$ is called the state space and contains all feasible states of the economy.

The technology of the economy is described by the transition possibility set $\Omega \subseteq X \times X$ and by the one-period return function $R: \Omega \rightarrow \mathbb{R}_{+}$. The transition possibility set $\Omega$ contains all pairs $(x, y) \in X \times X$ such that it is feasible to produce the capital stocks $y$ within one period if the vector of capital stocks at the beginning of that period was $x$. For each $x \in X$, we call $\Omega_{x}=\{y \in X \mid(x, y) \in \Omega\}$ the $x$-section of $\Omega$, and we assume that $\Omega_{x}$ is non-empty for all $x \in X$. The value of the one-period return function, $R(x, y)$, denotes the amount of output that is available for consumption in a given period if the vector of capital 
stocks at the beginning of that period is $x$ and if the capital stocks at the end of the period are restricted to be $y$. A path of capital stocks $\left(x_{t-1}\right)_{t=1}^{\infty}$ is feasible if it satisfies $x_{t} \in \Omega_{x_{t-1}}$ for all $t \in \mathbb{N}$.

The economy is populated by $n$ players, where $n \in \mathbb{N}$. All players are infinitely lived and identical. We denote by $c_{t}^{i}$ player $i$ 's consumption level in period $t$. Given a feasible path $\left(x_{t-1}\right)_{t=1}^{\infty}$, the vector of individual consumption levels $\left(c_{t}^{1}, c_{t}^{2}, \ldots, c_{t}^{n}\right) \in \mathbb{R}_{+}^{n}$ is feasible in period $t$ if it satisfies $\sum_{i=1}^{n} c_{t}^{i} \leq$ $R\left(x_{t-1}, x_{t}\right)$. Player $i \in\{1,2, \ldots, n\}$ seeks to maximize the objective functional

$$
\sum_{t=1}^{\infty} \rho^{t-1} u\left(c_{t}^{i}, x_{t-1}\right)
$$

where $\rho \in(0,1)$ is the discount factor and $u: \mathbb{R}_{+} \times X \rightarrow \mathbb{R}$ is the one-period utility function.

The game under consideration is described by the fundamentals $(n, X, \Omega, R, u, \rho)$. It is a simultaneousmove game with perfect information. Each player $i$ adopts a stationary Markovian strategy $\sigma^{i}: X \rightarrow \mathbb{R}_{+}$ that determines the individual consumption level in each period $t$ as a function of the stock level at the beginning of that period, that is $c_{t}^{i}=\sigma^{i}\left(x_{t-1}\right)$. A strategy profile $\left(\sigma^{1}, \sigma^{2}, \ldots, \sigma^{n}\right)$ is feasible if, for any initial stock $x \in X$, there is a feasible path $\left(x_{t-1}\right)_{t=1}^{\infty}$ that satisfies $x_{0}=x$ and $\sum_{i=1}^{n} \sigma^{i}\left(x_{t-1}\right) \leq R\left(x_{t-1}, x_{t}\right)$ for all $t \in \mathbb{N}$.

A strategy profile $\left(\sigma^{1}, \sigma^{2}, \ldots, \sigma^{n}\right)$ is a Markov-perfect Nash equilibrium (MPNE) if it is feasible and if, for each possible initial stock level $x \in X$ and for each player $i$, the dynamic optimization problem

$$
\begin{gathered}
\max _{\left(c_{t}^{i}, x_{t-1}^{i}\right)_{t=1}^{\infty}}\left\{\liminf _{T \rightarrow \infty} \sum_{t=1}^{T} \rho^{t-1} u\left(c_{t}^{i}, x_{t-1}^{i}\right)\right\} \\
\text { subject to }\left(x_{t-1}^{i}, x_{t}^{i}\right) \in \Omega \text { for all } t \in \mathbb{N},
\end{gathered}
$$

$$
\begin{aligned}
& R\left(x_{t-1}^{i}, x_{t}^{i}\right)-\sum_{j \neq i} \sigma^{j}\left(x_{t-1}^{i}\right)-c_{t}^{i} \geq 0 \text { for all } t \in \mathbb{N}, \\
& x_{0}^{i}=x
\end{aligned}
$$

has a unique solution $\left(c_{t}^{i *}, x_{t-1}^{i *}\right)_{t=1}^{\infty}$ that satisfies the following two conditions:

1. $c_{t}^{i *}=\sigma^{i}\left(x_{t-1}^{i *}\right)$ for all $t \in \mathbb{N}$.

2. There exists a feasible path $\left(x_{t-1}\right)_{t=1}^{\infty}$ with initial state $x_{0}=x$ such that $x_{t}^{i *}=x_{t}$ holds for all 
$i \in\{1,2, \ldots, n\}$ and all $t \in \mathbb{N}$.

We call the feasible path $\left(x_{t-1}\right)_{t=1}^{\infty}$ mentioned in condition 2 above the MPNE path corresponding to the MPNE $\left(\sigma^{1}, \sigma^{2}, \ldots, \sigma^{n}\right)$. We say that the MPNE $\left(\sigma^{1}, \sigma^{2}, \ldots, \sigma^{n}\right)$ is symmetric, if there exists a function $\sigma: X \rightarrow \mathbb{R}_{+}$such that $\sigma^{i}=\sigma$ holds for all $i \in\{1,2, \ldots, n\}$.

\subsection{Dynamic optimization problems}

We call the following problem the dynamic optimization problem $(X, \Omega, R, \rho)$ with initial state $x$ :

$$
\begin{gathered}
\max _{\left(x_{t-1}\right)_{t=1}^{\infty}}\left\{\sum_{t=1}^{\infty} \rho^{t-1} R\left(x_{t-1}, x_{t}\right)\right\} \\
\text { subject to }\left(x_{t-1}, x_{t}\right) \in \Omega \text { for all } t \in \mathbb{N}, \\
x_{0}=x .
\end{gathered}
$$

Here, $x$ is an arbitrary but fixed element of $X$. Let us denote by $V: X \rightarrow \mathbb{R}_{+} \cup\{\infty\}$ the optimal value function of $(X, \Omega, R, \rho)$. It is known that this function satisfies the Bellman equation

$$
V(x)=\max _{y}\{R(x, y)+\rho V(y) \mid(x, y) \in \Omega\}
$$

If it is true for all $x \in X$ that the problem on the right-hand side of (2.3) has a unique solution, say, $y=h(x)$, then we call the function $h: X \rightarrow X$ defined in that way the optimal policy function of $(X, \Omega, R, \rho)$.

\subsection{Rationalizability}

We say that a function $F: X \rightarrow X$ is rationalized by a dynamic game $(n, X, \Omega, R, u, \rho)$ if, for all $x_{0} \in X$, it holds that the game $(n, X, \Omega, R, u, \rho)$ with initial state $x_{0}$ has a MPNE path $\left(x_{t-1}\right)_{t=1}^{\infty}$ that satisfies the difference equation $x_{t}=F\left(x_{t-1}\right)$. Analogously, we say that $F$ is rationalized by a dynamic optimization problem $(X, \Omega, R, \rho)$ if $F$ is the optimal policy function of that problem. We will be particularly interested in conditions ensuring that the optimal policy function of a dynamic optimization problem can be rationalized by a dynamic game with $n \geq 2$ players. We shall also derive conditions under which the dynamics 
generated by a MPNE of a game with $n$ players can be rationalized by a dynamic optimization problem of a single decision maker.

\section{Rationalizability by a dynamic game}

Let us consider a dynamic optimization problem $(X, \Omega, R, \rho)$ satisfying the following assumption A1.

A1: For all $x \in X$ it holds that $V(x)<\infty$ and that the unique maximizer of the right-hand side of $(2.3)$ is given by $y=h(x)$, where $h: X \rightarrow X$ is a given function.

Note that assumption A1 implies that $h$ is the optimal policy function of $(X, \Omega, R, \rho)$. Assumption A1 can also be formulated as the requirement that for all $(x, y) \in \Omega$ with $y \neq h(x)$ it holds that

$$
\infty>V(x)=R(x, h(x))+\rho V(h(x))>R(x, y)+\rho V(y) .
$$

Moreover, since $R(x, y) \geq 0$ for all $(x, y) \in \Omega$, it follows that $V(x)$ is non-negative for all $x \in X$. Finally, the assumption $V(x)<\infty$ implies that

$$
\lim _{t \rightarrow \infty} \rho^{t} V\left(h^{t}(x)\right)=0 \text { for all } x \in X .
$$

This is the case because

$$
\infty>V(x)=\sum_{t=1}^{\infty} \rho^{t-1} R\left(h^{t-1}(x), h^{t}(x)\right)=\lim _{T \rightarrow \infty}\left(\sum_{t=1}^{T} \rho^{t-1} R\left(h^{t-1}(x), h^{t}(x)\right)+\rho^{T} V\left(h^{T}(x)\right)\right) .
$$

We are now ready to state our first result.

Lemma 3.1 Let $h: X \rightarrow X$ be a given function and let $(X, \Omega, R, \rho)$ be an optimization problem satisfying assumption $A 1$ (i.e., $h$ is the optimal policy function of $(X, \Omega, R, \rho)$ ). Furthermore, let $n \geq 2$ be an arbitrary integer and assume that there exists a function u satisfying the following assumptions $P_{u} 1-P_{u} 3$ : $P_{u}$ 1: For all $x \in X$ it holds that $u(c, x)$ is a strictly increasing and concave function of $c$.

$P_{u}$ 2: For all $x \in X, \partial u\left(n^{-1} R(x, h(x)), x\right) / \partial c$ exists and satisfies $\partial u\left(n^{-1} R(x, h(x)), x\right) / \partial c=n^{-1}$. $P_{u}$ 3: For all $x \in X$ it holds that $u\left(n^{-1} R(x, h(x)), x\right)=n^{-1} R(x, h(x))$. 
Then, it follows that $h$ is rationalized by $(n, X, \Omega, R, u, \rho)$. More specifically, $h$ can be rationalized by a symmetric $\operatorname{MPNE}(\sigma, \sigma, \ldots, \sigma)$ with $\sigma(x)=n^{-1} R(x, h(x))$.

Proof. Suppose that the one-period utility function $u$ satisfies assumptions $\mathrm{P}_{u} 1-\mathrm{P}_{u} 3$ and that all players other than player $i$ use the Markovian strategy $\sigma(x)=n^{-1} R(x, h(x))$. Then, player $i$ 's problem is written as

$$
\max _{\left(x_{t-1}\right)_{t=1}^{\infty}}\left\{\liminf _{T \rightarrow \infty} \sum_{t=1}^{T} \rho^{t-1} u\left(R\left(x_{t-1}, x_{t}\right)-(n-1) \sigma\left(x_{t-1}\right), x_{t-1}\right)\right\}
$$

subject to $\left(x_{t-1}, x_{t}\right) \in \Omega$ for all $t \in \mathbb{N}$,

$$
R\left(x_{t-1}, x_{t}\right)-(n-1) \sigma\left(x_{t-1}\right) \geq 0 \text { for all } t \in \mathbb{N}
$$

$x_{0}$ given.

If $h$ is the optimal policy function for this problem, then it follows that $\sigma$ is the unique best response of player $i$, because

$$
R\left(x_{t-1}, h\left(x_{t-1}\right)\right)-(n-1) \sigma\left(x_{t-1}\right)=n^{-1} R\left(x_{t-1}, h\left(x_{t-1}\right)\right)=\sigma\left(x_{t-1}\right) .
$$

This, in turn, implies that $(\sigma, \sigma, \ldots, \sigma)$ is a MPNE and that $h$ is rationalized by the dynamic game $(n, X, \Omega, R, u, \rho)$. Therefore, all we need to show is that $h$ is the optimal policy function of problem (3.3).

To this end note that $\mathrm{P}_{u} 1, \mathrm{P}_{u} 2$, and (3.1) imply for all $(x, y) \in \Omega$ that

$$
\begin{aligned}
& u(R(x, h(x))-(n-1) \sigma(x), x)-u(R(x, y)-(n-1) \sigma(x), x) \\
& \geq\{\partial u(R(x, h(x))-(n-1) \sigma(x), x) / \partial c\}[R(x, h(x))-R(x, y)] \\
& =n^{-1}[R(x, h(x))-R(x, y)] \\
& \geq n^{-1}[\rho V(y)-\rho V(h(x))]
\end{aligned}
$$

Therefore, for all $(x, y) \in \Omega$ it holds that

$$
u(R(x, h(x))-(n-1) \sigma(x), x)+n^{-1} \rho V(h(x)) \geq u(R(x, y)-(n-1) \sigma(x), x)+n^{-1} \rho V(y) .
$$


Next, we note that assumption $\mathrm{P}_{u} 3$ and the definition of $\sigma$ imply that

$$
u(R(x, h(x))-(n-1) \sigma(x), x)=n^{-1} R(x, h(x))
$$

It follows from this equality together with (3.1) and (3.4) that

$$
n^{-1} V(x)=\max _{y}\left\{u(R(x, y)-(n-1) \sigma(x), x)+\rho n^{-1} V(y) \mid(x, y) \in \Omega\right\}
$$

holds for all $x \in X$. Equation (3.6) shows that, for any feasible path $\left(x_{t-1}\right)_{t=1}^{\infty}$ starting from $x_{0}=x$, the inequality

$$
\begin{aligned}
& \sum_{t=1}^{T} \rho^{t-1} u\left(R\left(h^{t-1}(x), h^{t}(x)\right)-(n-1) \sigma\left(h^{t-1}(x)\right), h^{t-1}(x)\right) \\
&-\sum_{t=1}^{T} \rho^{t-1} u\left(R\left(x_{t-1}, x_{t}\right)-(n-1) \sigma\left(x_{t-1}\right), x_{t-1}\right) \geq \rho^{T} n^{-1} V\left(x_{T}\right)-\rho^{T} n^{-1} V\left(h^{T}(x)\right)
\end{aligned}
$$

must be satisfied. For expositional convenience we define

$$
a_{T}=\sum_{t=1}^{T} \rho^{t-1} u\left(R\left(h^{t-1}(x), h^{t}(x)\right)-(n-1) \sigma\left(h^{t-1}(x)\right), h^{t-1}(x)\right)
$$

and

$$
b_{T}=\sum_{t=1}^{T} \rho^{t-1} u\left(R\left(x_{t-1}, x_{t}\right)-(n-1) \sigma\left(x_{t-1}\right), x_{t-1}\right),
$$

and we note that

$$
a_{T}=\sum_{t=1}^{T} \rho^{t-1} n^{-1} R\left(h^{t-1}(x), h^{t}(x)\right)
$$

holds because of (3.5). Furthermore, $\lim _{T \rightarrow \infty} a_{T}$ exists and condition (3.7) implies

$$
\lim _{T \rightarrow \infty} a_{T}-\liminf _{T \rightarrow \infty} b_{T} \geq \limsup _{T \rightarrow \infty}\left(a_{T}-b_{T}\right) \geq \limsup _{T \rightarrow \infty}\left[\rho^{T} n^{-1} V\left(x_{T}\right)-\rho^{T} n^{-1} V\left(h^{T}(x)\right)\right] \geq 0 .
$$

The last inequality follows from the non-negativity of $V$ and from (3.2). Therefore, we have proved that the path $\left(h^{t}(x)\right)_{t=0}^{\infty}$ is an optimal path for problem (3.3) starting from the initial state $x$. We have also 
shown that the optimal value function of that problem is $n^{-1} V(x)$ and that the corresponding Bellman equation is (3.6). It remains to be shown that the right-hand side of (3.6) has a unique maximizer. To this end note that (3.1) implies that (3.4) holds with strict inequality if $y \neq h(x)$. But this implies obviously that the unique maximizer on the right-hand side of (3.6) is given by $h(x)$ which completes the proof of the lemma.

Remark. A key point in the above proof is that the solution of the functional equation (3.6) is indeed the optimal value function of the optimization problem (3.3). In order to ensure this property, the nonnegativity of the return function $R$ has been utilized in the proof. We can drop the condition $R \geq 0$ if we make some alternative assumptions. For example, one could assume that, for any feasible path $\left(x_{t-1}\right)_{t=1}^{\infty}$,

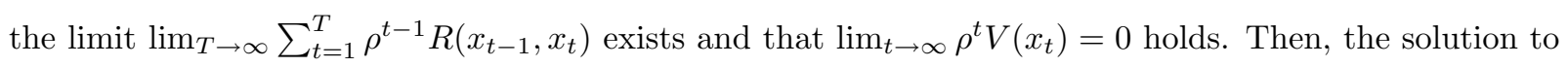
the functional equation (3.6) is the optimal value function of the optimization problem (3.3); see Stokey and Lucas (1989, Theorem 4.3). Alternatively, one could assume (i) that the transition possibility set $\Omega$ is convex, has non-empty interior, and satisfies $\Omega_{x} \subseteq \Omega_{y}$ for all $x, y \in X$ such that $x \leq y$, (ii) that the return function $R(x, y)$ is concave and increasing with respect to $x$, and (iii) that the transversality condition $\lim _{t \rightarrow \infty} \rho^{t} p_{t} h^{t}(x)=0$ holds, where $p_{t} \in \partial V\left(h^{t}(x)\right) \subseteq \mathbb{R}_{+}^{m}$ and $\partial V(x)$ is the subdifferential of $V$ at $x \in X$. With these assumptions the optimal value function $V(x)$ is concave. Also, the one-period utility function $u(R(x, y)-(n-1) \sigma(x), x)$ in (3.6) is concave in $y$. Then, the Bellman equation (3.6) implies that for each $t \in \mathbb{N}$ and for all $(x, y) \in \Omega$ it holds that

$$
\begin{aligned}
u\left(R\left(h^{t-1}(x), h^{t}(x)\right)-(n-1) \sigma\left(h^{t-1}(x)\right), h^{t-1}(x)\right)-n^{-1} p_{t-1} h^{t-1}(x)+\rho n^{-1} p_{t} h^{t}(x) \\
\geq u(R(x, y)-(n-1) \sigma(x), x)-n^{-1} p_{t-1} x+\rho n^{-1} p_{t} y
\end{aligned}
$$

see, for example, McKenzie (1986). This inequality and the transversality condition lead to the same result as (3.8), namely

$$
\lim _{T \rightarrow \infty} a_{T}-\liminf _{T \rightarrow \infty} b_{T} \geq \limsup _{T \rightarrow \infty} \rho^{T} n^{-1} p_{T}\left[x_{T}-h^{T}(x)\right] \geq \limsup _{T \rightarrow \infty} \rho^{T} n^{-1} p_{T} x_{T} \geq 0 .
$$

An obvious question that arises from Lemma 3.1 is whether there exists a utility function $u$ satisfying 
$\mathrm{P}_{u} 1-\mathrm{P}_{u} 3$. The next lemma proves that this is indeed the case.

Lemma 3.2 The following functions satisfy assumptions $P_{u} 1-P_{u} 3$ in Lemma 3.1:

$$
u(c, x)=c^{\frac{1}{n}}\left[n^{-1} R(x, h(x))\right]^{1-\frac{1}{n}}
$$

and

$$
u(c, x)=\frac{1}{n} c+\frac{n-1}{n}\left[n^{-1} R(x, h(x))\right]
$$

Putting together Lemmas 3.1 and 3.2 we obtain the main result of this section.

Theorem 3.1 Let $h: X \rightarrow X$ be a given function and let $(X, \Omega, R, \rho)$ be an optimization problem satisfying assumption $A 1$ (i.e., $h$ is the optimal policy function of $(X, \Omega, R, \rho)$ ). For any $n \geq 2$ there exists a dynamic game $(n, X, \Omega, R, u, \rho)$ that rationalizes $h$ through a symmetric MPNE. Here, the utility function $u$ can be chosen to satisfy the properties $P_{u} 1-P_{u} 3$ in Lemma 3.1.

We conclude this section by pointing out a corollary of the above result. Mitra and Sorger (1999) have shown that every Lipschitz continuous function $F: X \rightarrow X$ defined on a compact and convex domain $X \subseteq \mathbb{R}^{m}$ with nonempty interior can be rationalized by a dynamic optimization problem $(X, \Omega, R, \rho)$ provided that $\rho$ is chosen smaller than $1 / L^{2}$, where $L$ is a Lipschitz constant for $F$ (see Theorem 3 in Mitra and Sorger, 1999). Furthermore, they have shown that the problem $(X, \Omega, R, \rho)$ can be chosen such that the following properties hold:

$\mathrm{P}_{\Omega}: \Omega \subseteq X \times X$ is a closed and convex set such that the $x$-section $\Omega_{x}$ is nonempty for all $x \in X$. The set $\bigcup_{x \in X} \Omega_{x}$ has a nonempty interior. If $x \leq \bar{x}$, then $\Omega_{x} \subseteq \Omega_{\bar{x}}$.

$\mathrm{P}_{R}: R: \Omega \rightarrow \mathbb{R}$ is a continuous and concave function. $R(x, y)$ is nondecreasing in $x$ and nonincreasing in $y$.

$\mathrm{P}_{V}$ : The optimal value function $V$ is $\alpha$-concave and $(-\beta)$-convex for positive numbers $\alpha$ and $\beta$.

By combining this result with Theorem 3.1 one obtains the following corollary.

Corollary 3.2 Let $X \subseteq \mathbb{R}^{m}$ be a compact and convex set with a nonempty interior and let $F: X \rightarrow X$ be a Lipschitz continuous function with Lipschitz constant L. Furthermore, let $\rho \in\left(0,1 / L^{2}\right)$ and $n \in \mathbb{N}$ 
with $n \geq 2$ be arbitrarily given. Then it follows that $F$ can be rationalized by a symmetric MPNE of a dynamic game of the form $(n, X, \Omega, R, u, \rho)$. Moreover, this game can be chosen such that the properties $P_{u} 1-3, P_{\Omega}, P_{R}$, and $P_{V}$ hold.

Proof. By the cited theorem from Mitra and Sorger (1999), the function $F$ is rationalized by a dynamic optimization problem $(X, \Omega, R, \rho)$ with optimal value function $V$. We only have to show that assumption A1 holds. The concavity of $R$ (property $\mathrm{P}_{R}$ ) and the strict concavity of $V$ (property $\mathrm{P}_{V}$ ) ensure that the right-hand side of the Bellman equation is maximized at the unique state $y=F(x)$. The compactness of $X$ and the closedness of $\Omega$ (Property $\mathrm{P}_{\Omega}$ ) imply that $\Omega$ is compact. Together with continuity of $V$ this implies that $V$ is bounded. Hence, assumption A1 holds and the corollary follows from Theorem 3.1.

\section{Properties of the utility function}

In this section, we discuss some properties of the utility function $u(c, x)$. Note that all the assumptions that we have imposed on the function $u$ in Lemma 3.1 regard its dependence on $c$. The dependence of $u$ on $x$ (the wealth effect) is completely unrestricted. If we have a dynamic game with a utility function that is increasing and concave jointly in $c$ and $x$, the model is of course more appealing from the viewpoint of economics.

We shall now derive conditions under which these properties can be ensured. To this end we first note that the utility functions defined in Lemma 3.2 are increasing and concave in $(c, x)$ if the mapping $x \rightarrow R(x, h(x))$ is increasing and concave. From the Bellman equation $(2.3), R(x, h(x))$ satisfies

$$
R(x, h(x))=V(x)-\rho V(h(x))
$$

These observations allow us to prove the following result.

Theorem 4.1 Let $X \subseteq \mathbb{R}^{m}$ be a compact and convex set with a nonempty interior and let $h: X \rightarrow X$ be a twice continuously differentiable function. Furthermore, let $n \in \mathbb{N}$ be given such that $n \geq 2$. Then it follows that $h$ can be rationalized by a symmetric MPNE of a dynamic game $(n, X, \Omega, R, u, \rho)$ with an increasing and concave utility function $u$ whenever $\rho$ is sufficiently small. 
Proof. Compactness of $X$ and twice continuous differentiability of $h$ imply that $h$ is Lipschitz continuous and, hence, that Theorem 3 in Mitra and Sorger (1999) is applicable. It follows that $h$ can be rationalized by a dynamic optimization problem $(X, \Omega, R, \rho)$. Moreover, in the proof of Theorem 3 in Mitra and Sorger (1999) it is shown that the the optimal value function of $(X, \Omega, R, \rho)$ can be chosen to be

$$
V(x)=\gamma e_{m} x-(\alpha / 2)\|x\|^{2},
$$

where $\alpha$ and $\gamma$ are positive real numbers and where $e_{m}=(1,1, \ldots, 1) \in \mathbb{R}^{m}$. Note that this function $V$ is strictly concave, and that it is strictly increasing if $\gamma$ is sufficiently large. Since $R(x, h(x))$ in (4.1) is twice continuously differentiable when $V(x)$ is given by the above formula, the first and second order derivatives uniformly converge to those of $V(x)$ as $\rho$ approaches 0 . Therefore, by choosing a sufficiently small value for $\rho, R(x, h(x))$ is increasing and concave. Using such $R(x, h(x))$ in the utility functions from Lemma 3.2 we obtain from Theorem 3.1 a dynamic game with an increasing and concave utility function.

We now discuss a few examples in order to illustrate the issues discussed above.

Example 1. (Neoclassical growth model) The so-called Brock-Mirman neoclassical growth model (Brock and Mirman, 1972) allows for a closed form solution for the optimal policy function. The optimization problem $(X, \Omega, R, \rho)$ is characterized by $X=[0,1], \Omega=\left\{(x, y) \in X \times X \mid 0 \leq y \leq x^{\alpha}\right\}$ with $\alpha \in(0,1)$, $R(x, y)=\ln \left(x^{\alpha}-y\right)$, and $\rho \in(0,1)$. The optimal policy function and the optimal value function, respectively, are given by

$$
h(x)=\alpha \rho x^{\alpha} \text { and } V(x)=\frac{\alpha}{1-\alpha \rho} \ln x+\nu,
$$

where

$$
\nu=\left[\ln (1-\alpha \rho)+\frac{\alpha \rho \ln \alpha \rho}{1-\alpha \rho}\right](1-\rho)^{-1}
$$

(For the derivation, see Stokey and Lucas, 1989, Chapter 2.) Then, $R(x, h(x))$ is given by

$$
R(x, h(x))=\alpha \ln x+\ln (1-\alpha \rho)
$$

which is increasing and concave. It follows that Theorem 3.1 can be directly applied ${ }^{1}$ and that no further

\footnotetext{
${ }^{1}$ Although the return function is not bounded below, the second set of alternative assumptions in the remark below Lemma 3.1 is applicable.
} 
restriction of the discount factor $\rho$ is required to ensure that the utility function $u$ is increasing and concave.

Example 2. (Logistic map policy function) Boldrin and Montrucchio (1986) show that any twice continuously differentiable function is rationalized by a dynamic optimization problem $(X, \Omega, R, \rho)$. They illustrate their result with the logistic map $h(x)=4 x(1-x)$, which generates chaotic dynamics. Let $X=[0,1]$ and $\Omega=X \times X$. According to Boldrin and Montrucchio (1986), the dynamical system $h$ is rationalized with the return function

$$
\begin{aligned}
R(x, y)=-(1 / 2)(3-2 \sqrt{2}) y^{4}+(3-2 \sqrt{2}) y^{3}- & (2 \sqrt{2}-5 / 2) y^{2} \\
& -(75 / 8)(3-2 \sqrt{2}) y+4 x(1-x) y-24 x^{2}+150 x,
\end{aligned}
$$

when the discount factor satisfies $\rho=(3-2 \sqrt{2}) / 16 \approx 0.01072$. This implies

$$
\begin{aligned}
R(x, h(x))=(75 / 2) & (2 \sqrt{2}+1) x-(107 \sqrt{2}-289 / 2) x^{2}-16(4 \sqrt{2}-5) x^{3}-8(113-76 \sqrt{2}) x^{4} \\
& +704(3-2 \sqrt{2}) x^{5}-832(3-2 \sqrt{2}) x^{6}+512(3-2 \sqrt{2}) x^{7}-128(3-2 \sqrt{2}) x^{8} .
\end{aligned}
$$

It is straightforward to verify that $R(x, h(x))$ is increasing and concave on $X$ and it follows again that Theorem 4.1 is not needed to ensure the monotonicity and concavity of $u$.

Example 3. (Tent map policy function) The last example fails to meet the requirement of concavity. Nishimura and Yano (1995) demonstrate that under standard assumptions in economics, even when the discount factor is sufficiently close to 1 , the optimal policy function may be expansive and unimodal, implying a chaotic equilibrium path. Their result is based on the following example. Let $\alpha, \beta, \mu$, and $\rho$ be positive real numbers such that $\alpha \in(\beta /(1+\mu), \beta / \mu), \mu / \beta<1, \rho \mu>1$, and $\rho<1$ and define $X=[0, \mu / \beta]$, $\Omega=\{(x, y) \in X \times X \mid x \leq \min \{\mu x, \mu / \beta\}\}$, and

$$
R(x, y)=\left\{\begin{array}{cc}
x-\mu^{-1} y & \text { if } 0 \leq y \leq-\frac{\alpha \mu}{\beta-\alpha} x+\frac{\mu}{\beta-\alpha} \\
\frac{1}{\alpha}-\frac{\beta / \alpha}{\mu} y & \text { otherwise. }
\end{array}\right.
$$

Nishimura and Yano (1995) show that the optimal policy function of the dynamic optimization problem 
$(X, \Omega, R, \rho)$ is given by

$$
h(x)=\left\{\begin{array}{cc}
\mu x & \text { if } 0 \leq x \leq 1 / \beta \\
-\frac{\alpha \mu}{\beta-\alpha} x+\frac{\mu}{\beta-\alpha} & \text { otherwise. }
\end{array}\right.
$$

This implies that

$$
R(x, h(x))=\left\{\begin{array}{cc}
0 & \text { if } 0 \leq x \leq 1 / \beta \\
\frac{\beta x-1}{\beta-\alpha} & \text { otherwise }
\end{array}\right.
$$

Hence, the return function evaluated at $y=h(x)$ is increasing but not concave due to the corner solutions of $h(x)$ on $[0,1 / \beta]$.

\section{A partial converse of Theorem 3.1}

The dynamic optimization problem $(X, \Omega, R, \rho)$ in $(2.2)$ can be interpreted as a special case of the dynamic game $(n, X, \Omega, R, u, \rho)$ with $n=1$ and $u(c, x)=c$. This implies trivially that every policy function of a dynamic optimization problem can be rationalized by a dynamic game. The strength of Theorem 3.1 derives from the fact that $n$ can be fixed at an arbitrary value greater than or equal to 2 and that symmetry of the equilibrium is imposed. On the other hand, it is not a trivial question at all whether the converse to Theorem 3.1 holds, that is, whether the dynamics generated by a dynamic game can be rationalized by an appropriately defined dynamic optimization problem. In this section, we prove at least a partial converse of Theorem 3.1. It is partial because we have to impose the additional assumptions A2 to A4; see below.

As in the previous sections, let $(n, X, \Omega, R, u, \rho)$ be a dynamic game and let $(\sigma, \sigma, \ldots, \sigma)$ with $\sigma: X \rightarrow$ $\mathbb{R}_{+}$be a symmetric MPNE of that game. As the counterpart to assumption A1 we make the following assumption.

A1': (a) The utility function $u: \mathbb{R}_{+} \times X \rightarrow \mathbb{R}_{+}$takes non-negative values and is strictly increasing and concave with respect to its first argument (i.e., with respect to consumption). 
(b) The equilibrium value function $v: X \rightarrow \mathbb{R}_{+}$defined by

$$
\begin{gathered}
\qquad(x)=\max _{\left(c_{t}, x_{t-1}\right)_{t=1}^{\infty}} \sum_{t=1}^{\infty} \rho^{t-1} u\left(c_{t}, x_{t-1}\right) \\
\text { subject to }\left(x_{t-1}, x_{t}\right) \in \Omega \text { for all } t \in \mathbb{N}, \\
R\left(x_{t-1}, x_{t}\right)-(n-1) \sigma\left(x_{t-1}\right)-c_{t} \geq 0 \text { for all } t \in \mathbb{N}, \\
x_{0}=x
\end{gathered}
$$

satisfies

$$
v(x)<\infty \text { for all } x \in X
$$

(c) For each $x \in X$ there is a unique value $h(x) \in \Omega_{x}$ such that

$$
v(x)=u(\sigma(x), x)+\rho v(h(x))=\max _{c \in \mathbb{R}_{+}, y \in \Omega_{x}}\{u(c, x)+\rho v(y) \mid R(x, y)-(n-1) \sigma(x)-c \geq 0\} .
$$

Assumption A1' is a straightforward adaptation of assumption A1, which was imposed on the dynamic optimization problem $(X, \Omega, R, \rho)$, to the framework of dynamic games. Note that the fact that $u$ is strictly increasing in $c$ implies that the inequality in (5.2) must hold as an equality if the consumption rate $c$ equals its equilibrium value $\sigma(x)$. This implies that

$$
\sigma(x)=n^{-1} R(x, h(x))
$$

Also note that

$$
v(x) \geq 0 \text { and } \lim _{t \rightarrow \infty} \rho^{t} v\left(h^{t}(x)\right)=0 \text { for all } x \in X,
$$

which follows from the non-negativity of the utility function and from (5.1)-(5.2).

In addition to A1' we make the following assumptions.

A2: $R: \Omega \rightarrow \mathbb{R}_{+}$is a concave function.

A3: It holds that $\Omega_{0}=\{0\}$ and $R(0,0)=0$. Moreover, $R(x, y)$ is increasing in $x$ and decreasing in $y$. If 
$x \neq 0$, then there exists $y \in X$ such that $R(x, y)>0$.

A4: $v: X \rightarrow \mathbb{R}_{+}$is a concave function.

While Assumptions A2 and A3 are quite innocuous standard assumptions, ${ }^{2}$ the concavity of $v$ in Assumption A4 is restrictive. Indeed, since Markov-perfect equilibrium strategies are typically non-linear functions of the state variables, the concavity of the equilibrium value functions can usually not be guaranteed even if the constraint sets are convex and all utility functions are concave. On the other hand, concavity of the optimal value function of a (single-player) dynamic optimization problem follows from convex constraint sets and concave utility functions. Since our approach is based on the construction of a dynamic optimization problem with the same value function as the dynamic game, we must assume that this function is concave. Technically speaking, the concavity of the equilibrium value function is adopted in order to apply the saddle point theorem.

Note that for the game problem (5.2) with $x \neq 0$, Slater's constraint qualification is satisfied: $R(x, y)-$ $(n-1) \sigma(x)-c$ is concave in $(c, y)$ and its value is strictly positive at some $(c, y)$ such that $0 \leq y \leq h(x)$ and $c \geq 0$. Therefore, by the saddle point theorem, for each $x \in X \backslash\{0\}$, there is a Lagrange multiplier $\lambda(x) \geq 0$ such that for all $\lambda \geq 0$, for all $c \geq 0$, and for all $y \in \Omega_{x}$ it holds that

$$
\begin{aligned}
& u(\sigma(x), x)+\rho v(h(x))+\lambda[R(x, h(x))-(n-1) \sigma(x)-\sigma(x)] \\
& \geq u(\sigma(x), x)+\rho v(h(x))+\lambda(x)[R(x, h(x))-(n-1) \sigma(x)-\sigma(x)] \\
& \geq u(c, x)+\rho v(y)+\lambda(x)[R(x, y)-(n-1) \sigma(x)-c] .
\end{aligned}
$$

When $x=0$, then assumption A3 implies that the feasible consumption and the capital stock at the end of the period are uniquely given by $(c, y)=(0,0)$. Therefore, the equilibrium strategy for this case must be $\sigma(0)=0$ and $h(0)=0$.

Now define the function $U: \mathbb{R}_{+} \times X \rightarrow \mathbb{R}$ by

$$
U(c, x)=u(c, x)+\lambda(x)(n-1)[c-\sigma(x))] .
$$

\footnotetext{
${ }^{2}$ These assumptions may be replaced with others as far as they ensure that the optimization problem in (5.2) is a convex one and that Slater's constraint qualification is satisfied.
} 
Also define the function $\tilde{R}: \Omega \rightarrow \mathbb{R}_{+}$by

$$
\tilde{R}(x, y)=U\left(n^{-1} R(x, y), x\right)
$$

We will show that the dynamical system $h$ that induces the MPNE path is rationalized by the dynamic optimization problem

$$
\begin{gathered}
\max _{\left(x_{t-1}\right)_{t=1}^{\infty}} \sum_{t=1}^{\infty} \rho^{t-1} \tilde{R}\left(x_{t-1}, x_{t}\right) \\
\text { subject to }\left(x_{t-1}, x_{t}\right) \in \Omega \text { for all } t \in \mathbb{N} \\
x_{0}=x .
\end{gathered}
$$

To this end, we prepare the following lemma.

Lemma 5.1 Under Assumptions A1' and A2-A4, the functional equation

$$
v(x)=U\left(n^{-1} R(x, h(x)), x\right)+\rho v(h(x))=\max _{y}\left\{U\left(n^{-1} R(x, y), x\right)+\rho v(y) \mid(x, y) \in \Omega\right\}
$$

holds.

Proof. For the case of $x=0$, condition (5.7) holds by assumption A3. Assume $x \in X \backslash\{0\}$. Note that $u(\sigma(x), x)=U(\sigma(x), x)$. Then, (5.5) implies that for $(\sigma(x), h(x))$ such that $\sigma(x) \geq 0, h(x) \in \Omega_{x}$, and $R(x, h(x))-\sigma(x) \geq 0$, there exists $\lambda(x)$ such that

$$
\begin{aligned}
& U(\sigma(x), x)+\rho v(h(x))+\lambda[R(x, h(x))-n \sigma(x)] \\
& \geq U(\sigma(x), x)+\rho v(h(x))+\lambda(x)[R(x, h(x))-n \sigma(x)] \\
& \geq U(c, x)+\rho v(y)+\lambda(x)[R(x, y)-n c] .
\end{aligned}
$$

Because the saddle point condition holds, we have for all $c \geq 0$ and all $y \in \Omega_{x}$ such that $R(x, y)-n c \geq 0$

$$
U(\sigma(x), x)+\rho v(h(x)) \geq U(c, x)+\rho v(y) .
$$


Finally, $u(\sigma(x), x)=U(\sigma(x), x)$ implies $v(x)=U(\sigma(x), x)+\rho v(h(x))$. Therefore, we have

$$
\begin{aligned}
v(x) & =U\left(n^{-1} R(x, h(x)), x\right)+\rho v(h(x)) \\
& =\max _{c, y}\left\{U(c, x)+\rho v(y) \mid c \geq 0, y \in \Omega_{x}, R(x, y)-n c \geq 0\right\},
\end{aligned}
$$

which is equivalent to $(5.7)$.

Theorem 5.1 Under Assumptions A1' and A2-A4, the function $h$ is rationalized by the optimization problem $(X, \Omega, \tilde{R}, \rho)$.

Proof. Using (5.6), one can rewrite (5.7) in Lemma 5.1 as

$$
v(x)=\tilde{R}(x, h(x))+\rho v(h(x)) \geq \tilde{R}(x, y)+\rho v(y) \text { for all }(x, y) \in \Omega .
$$

This implies that

$$
\sum_{t=1}^{\infty} \rho^{t-1} \tilde{R}\left(h^{t-1}(x), h^{t}(x)\right)-\liminf _{T \rightarrow \infty} \sum_{t=1}^{T} \rho^{t-1} \tilde{R}\left(x_{t-1}, x_{t}\right) \geq \limsup _{T \rightarrow \infty}\left[\rho^{T} v\left(x_{T}\right)-\rho^{T} v\left(h^{T}(x)\right)\right] \geq 0
$$

holds for all feasible path $\left(x_{t-1}\right)_{t=1}^{\infty}$ starting from $x_{0}=x$. Here, the last inequality follows from (5.4). Therefore, $\left(h^{t}(x)\right)_{t=0}^{\infty}$ is an optimal path for the optimization problem $(X, \Omega, \tilde{R}, \rho)$.

\section{Concluding remarks}

In this paper we have collected some results on the rationalizability of given dynamical systems by dynamic games and dynamic optimization problems. These results are meant to illustrate how large a set of dynamical systems is consistent with rational decision making by a single or by multiple decision makers. The results also give rise to the conjecture that a larger set of dynamical systems can be generated by MPNE of dynamic games than by optimal policy functions of single-player decision problems. A rigorous proof of this conjecture, however, is beyond the scope of the present paper.

In deriving our results, we have made a number of technical assumptions like the non-negativity of the one-period return functions in Section 3 or the utility functions in Section 5 . We do not think that 
these assumptions are crucial to our analysis and we have indicated at some places how to relax or replace them. Other assumptions, like those that ensure the uniqueness of the maximizers on the right-hand sides of the Bellman equations are essential to our arguments, as they ensure the existence of optimal policy functions without which most rationalizability results would be considerably weaker. In any case, we hope that the reader can appreciate the balance of assumptions that we have used in order to get interesting results and, at the same time, not to clutter the analysis with too many technicalities. 


\section{References}

[1] Boldrin, M., and Montrucchio, L., 1986: "On the indeterminacy of capital accumulation paths," Journal of Economic Theory 40, 26-39.

[2] Brock, W.A., and Mirman, L.J., 1972: "Optimal economic growth and uncertainty: the discounted case," Journal of Economic Theory 4, 479-513.

[3] Dana, R.-A., and Montrucchio, L., 1986: "Dynamic complexity in duopoly games," Journal of Economic Theory 40, 40-56.

[4] Deneckere, R., and Pelikan, S., 1986: "Competitive chaos," Journal of Economic Theory 40, 13-25.

[5] McKenzie, L.W., 1986: "Optimal economic growth, turnpike theorem and comparative dynamics," Handbook of Mathematical Economics 3 (eds.: Arrow, K.J., and Intriligator, M.D.), North-Holland, pp. 1281-1355.

[6] Mitra, T., and Sorger, G., 1999: "Rationalizing policy functions by dynamic optimization," Econometrica $67,375-392$.

[7] Nishimura, K., and Yano, M., 1995: "Nonlinear dynamics and chaos in optimal growth: an example," Econometrica 63, 981-1001.

[8] Sorger, G., 2006: "Rationalizability in optimal growth theory" Handbook on Optimal Growth 1 (eds.: Dana, R.-A., Le Van, C., Mitra, T., and Nishimura, K.), Springer-Verlag, pp. 85-113.

[9] Stokey, N.L., and Lucas, R.E., (with Prescott, E.C.) 1989: Recursive Methods in Economic Dynamics, Harvard University Press. 